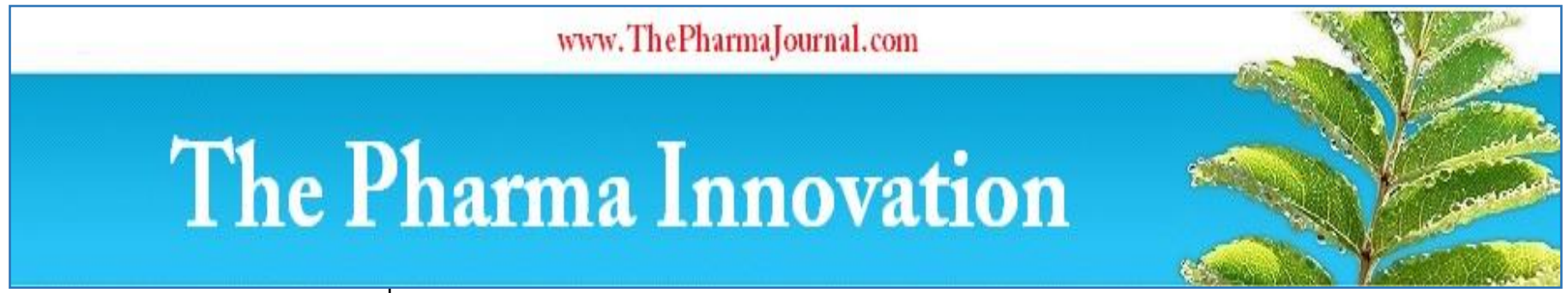

ISSN (E): 2277 - 7695

ISSN (P): 2349-8242

NAAS Rating: $\mathbf{5 . 0 3}$

TPI 2020; SP-9(10): 206-210

(C) 2020 TPI

www.thepharmajournal.com

Received: 06-08-2020

Accepted: 11-09-2020

\section{Srikala D}

Assistant Professor, SVVU Super Speciality Veterinary Hospital,

Visakhapatnam, Andhra

Pradesh, India

\section{Ansar Kamran}

Professor, Department of

Veterinary Medicine, Veterinary

College, Hebbal, KVAFSU,

Bangalore, Karnataka, India

\section{Nalini Kumari K}

Associate Dean, College of

Veterinary Science, SVVU,

Tirupati, Andhra Pradesh, India

\section{Ramesh PT}

Professor, Department of

Veterinary Medicine, Veterinary

College, Hebbal, KVAFSU,

Bangalore, Karnataka, India

\section{Suguna Rao}

Professor, Department of

Veterinary Pathology,

Veterinary College, Hebbal,

KVAFSU, Bangalore,

Karnataka, India

\section{Yathiraj S}

Retired Dean, Veterinary

College, Hebbal, KVAFSU,

Bangalore, Karnataka, India
Corresponding Author:

Srikala D

Assistant Professor, SVVU Super

Speciality Veterinary Hospital,

Visakhapatnam, Andhra

Pradesh, India

\section{A study on different clinical manifestations of geriatric dogs}

\author{
Srikala D, Ansar Kamran, Nalini Kumari K, Ramesh PT, Suguna Rao \\ and Yathiraj $S$
}

DOI: https://doi.org/10.22271/tpi.2020.v9.i9Se.5263

\section{Abstract}

There is a growing interest in health care and wellness of elderly dogs. initial clinical signs are vague and difficult to recognize for the owner or discarded as not important or "normal for his age. A total of 528 geriatric dogs including 82 male and 74 female that were presented to SVVU Super Speciality Veterinary Hospital, Visakhapatnam, with different ailments. most prominent clinical signs observed in geriatric dogs at presentation were white hair, calluses, loss of body weight, dehydration, hair loss, dull, dry hair coat, dental tartar, loss of tooth, panting, broken tooth, scaly skin etc., The clinical approach is an important tool to achieve effective intervention and better health management.

Keywords: Geriatric dogs, clinical signs, clinical manifestations

\section{Introduction}

There is a growing interest in health care and wellness of elderly dogs ${ }^{[1]}$. Geriatric group has specific needs and is more prone to develop chronic illness ${ }^{[2]}$. However, the scientific information regarding abnormalities on physical examination findings in this age group is limited. Elderly pets represent $30-40 \%$ of patients in general practice and this proportion is likely to increase in the future as dogs live longer ${ }^{[3]}$. The current study aimed to prospectively evaluate the clinical manifestations of geriatric dogs that were sick and to report on the presence of abnormal findings on physical examination. Geriatric dogs has specific needs and is more prone to develop chronic illness ${ }^{[2]}$ often, initial clinical signs are vague and difficult to recognize for the owner or discarded as not important or "normal for his age ${ }^{[4]}$. The goal of these programs is to improve quality of life and longevity, through early detection and timely treatment of diseases.

\section{Materials and methods}

The present clinical research included 528 geriatric dogs including 82 male and 74 female that were presented to SVVU Super Speciality Veterinary Hospital, Visakhapatnam, with a history of going down in condition with generalised weakness, poor physical activity, chronic anorexia and fluid accumulation at various parts of the body and signs of respiratory distress, abnormal mucosa, lameness, edema of dependent parts, polyuria, polydypsia/oliguria, difficulty in sitting down and getting up, during the period from January 2016 to May 2019. These cases were subjected for detailed history and physical examination.

\section{Results and Discussion}

In the present study most prominent clinical signs observed in geriatric dogs at presentation were white hair $(92.19 \%)$, calluses $(76.39 \%)$, loss of body weight $(71.38 \%)$, dehydration (71.19\%), hair loss $(68.22 \%)$, dull, dry hair coat $(66.36 \%)$, dental tartar $(60.59 \%)$, loss of tooth $(58.36 \%)$, panting $(50.93 \%)$, broken tooth $(49.26 \%)$, scaly skin $(46.65 \%)$, hyperkeratosis $(43.31 \%)$, constipation $(39.22 \%)$, subcutaneous mass $(36.99 \%)$, loss of vision $(34.39 \%)$, cutaneous neoplasia (34.57\%), plaque (29.00\%), diarrhea (31.23\%), loss of hearing $(27.32 \%)$, obesity $(23.79 \%)$ and pendulous, potbelly abdomen $(23.42 \%)$ was given in the Table. 1 and depicted in Fig. 1. 
Table 1: Clinical manifestations noticed in sick geriatric dogs $(\mathrm{n}=538)$

\begin{tabular}{|c|c|c|c|}
\hline Sl. No. & Clinical signs & No. of dogs & Per cent \\
\hline 1 & White hair & 496 & 92.19 \\
\hline 2 & Calluses & 411 & 76.39 \\
\hline 3 & Loss of body weight & 384 & 71.38 \\
\hline 4 & Dehydration & 383 & 71.19 \\
\hline 5 & Hair loss & 367 & 68.22 \\
\hline 6 & Dull, dry hair coat & 357 & 66.36 \\
\hline 7 & Dental tartar & 326 & 60.59 \\
\hline 8 & Loss of tooth & 314 & 58.36 \\
\hline 9 & Panting & 274 & 50.93 \\
\hline 10 & Broken tooth & 265 & 49.26 \\
\hline 11 & Scaly skin & 46.65 \\
\hline 12 & Hyperkeratosis & 233 & 43.31 \\
\hline 13 & Constipation & 199 & 39.22 \\
\hline 14 & Subcutaneous mass & 186 & 36.99 \\
\hline 15 & Cutaneous neoplasia & 185 & 34.57 \\
\hline 16 & Loss of vision & 170 & 31.59 \\
\hline 17 & Halitosis & 168 & 31.23 \\
\hline 18 & Diarrhoea & 156 & 29.00 \\
\hline 19 & Plaque & 147 & 27.32 \\
\hline 20 & Loss of hearing & 128 & 23.79 \\
\hline 21 & Obesity & 23.42 \\
\hline 22 & Pendulous, potbelly abdomen & 126 & \\
\hline
\end{tabular}

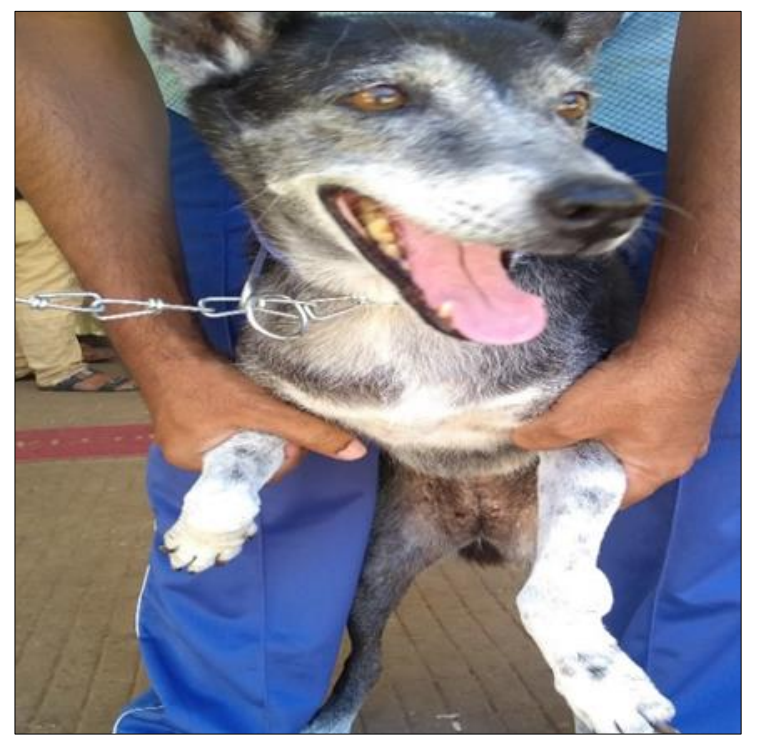

Fig 1: Greying of hair around face and muzzle in 11-year-old nondescript geriatric dog

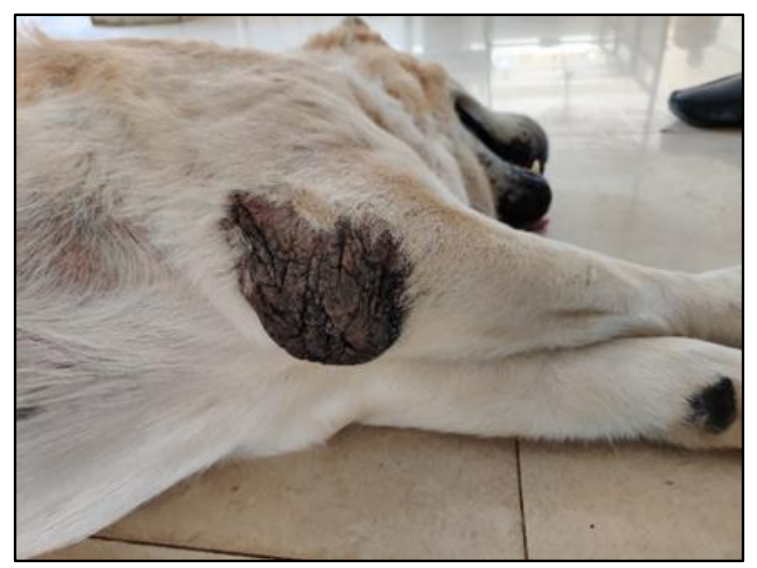

Fig 2: Calluses formation at the elbow joint of 12-year-old Labrador Retriever

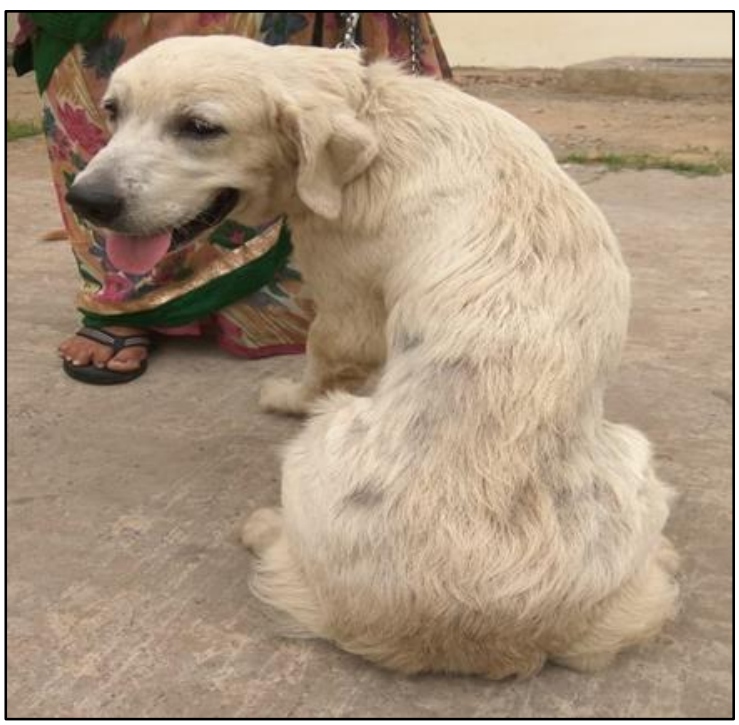

Fig 3: Loss of body weight in a 12-year-old Labrador Retriever

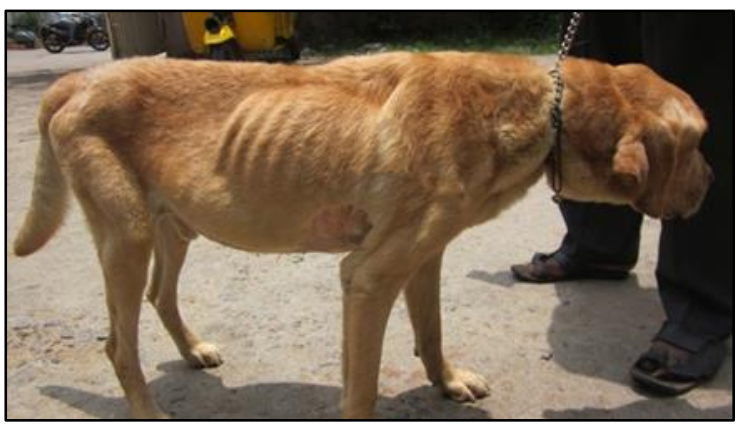

Fig 4: Dehydration in 10-year-old mixed breed dog

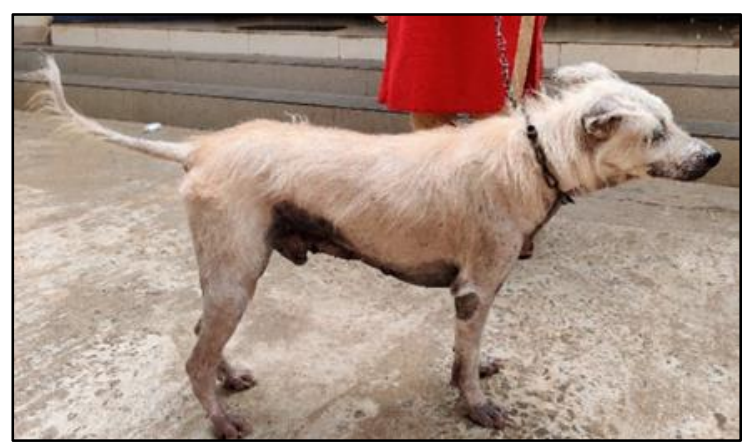

Fig 5: Loss of hair in 9-year-old Spitz

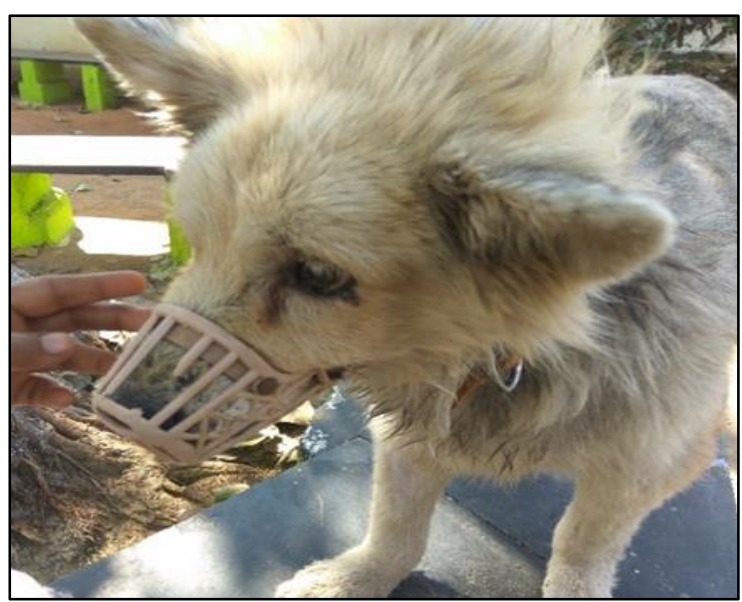

Fig 6: Dry dull hair in 9-year-old dog 


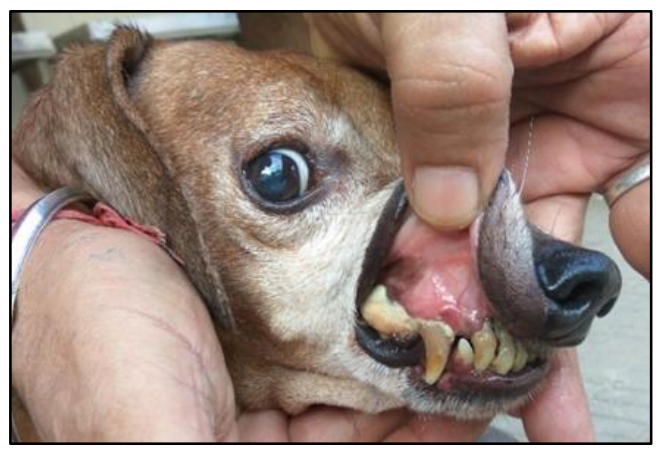

Fig 7: Dental tartar in a 12-year-old Labrador Retriever dog

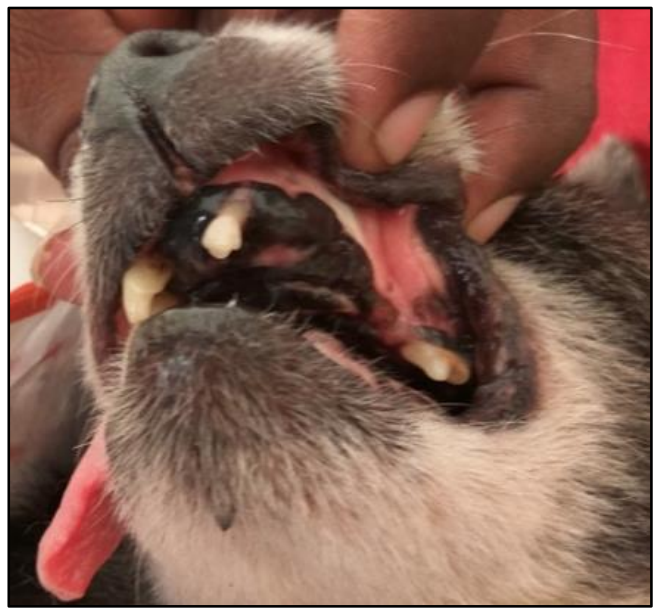

Fig 8: Loss of tooth in a 14-year-old mixed breed dog

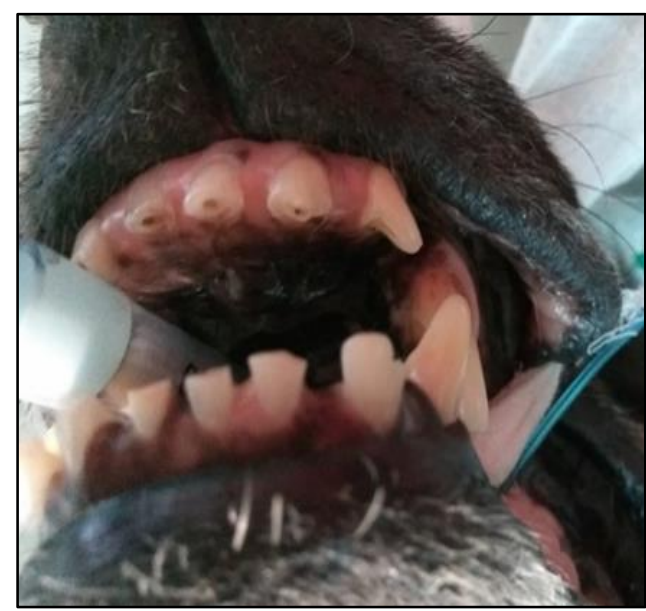

Fig 9: Broken tooth in 12-year-old dog

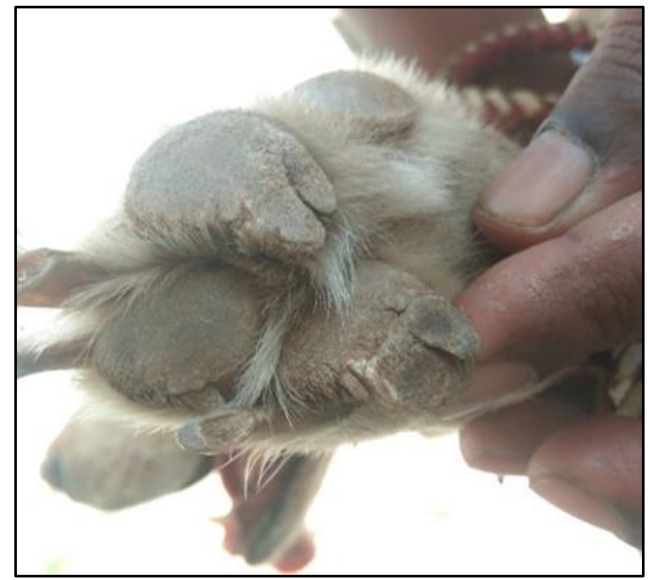

Fig 10: Hyperkeratosis or thickening of foot pad in geriatric dog

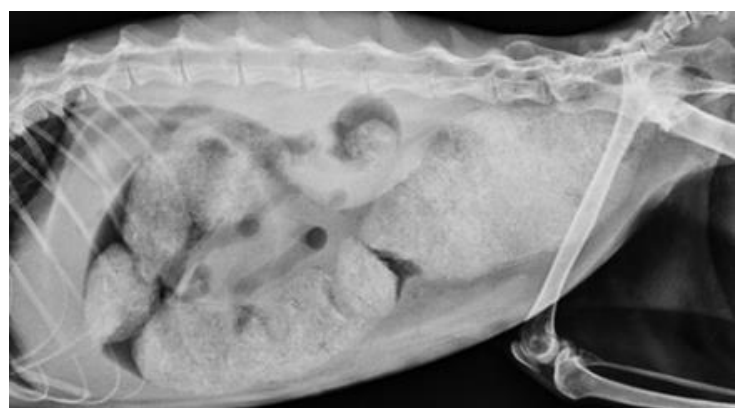

Fig 11: Radiograph of lateral abdomen showing severe constipation

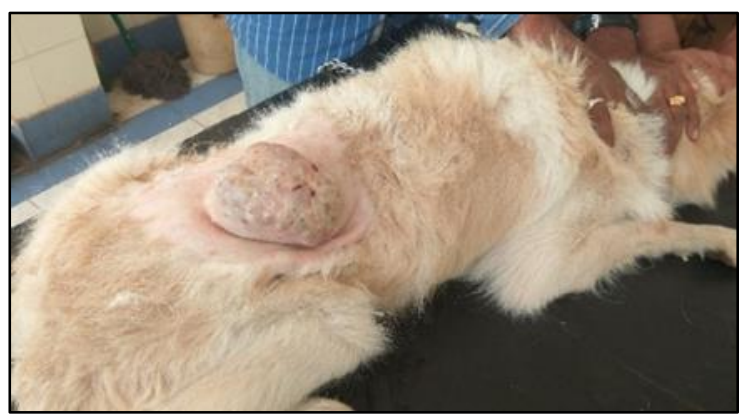

Fig 12: Subcutaneous masses in a geriatric dog

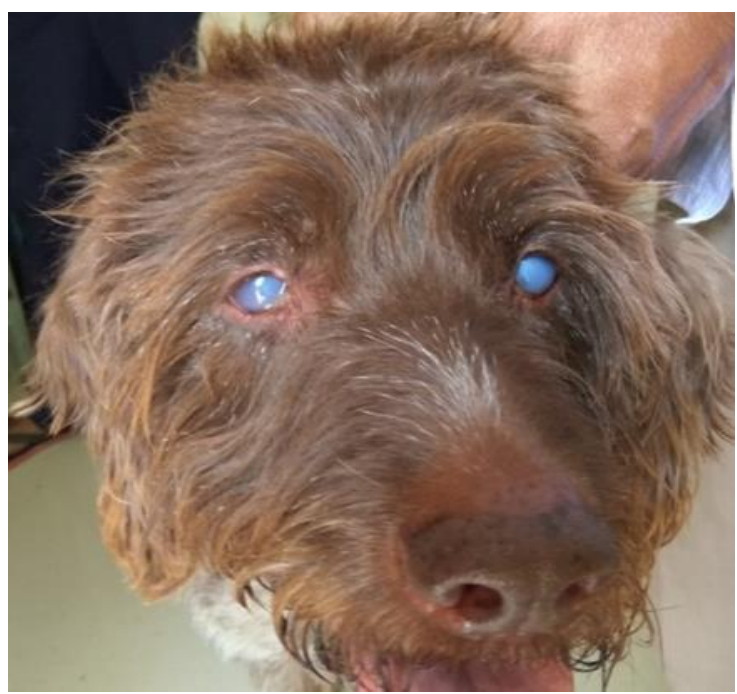

Fig 13: Loss of vision in a 10-year-old dog due to bilateral cataract

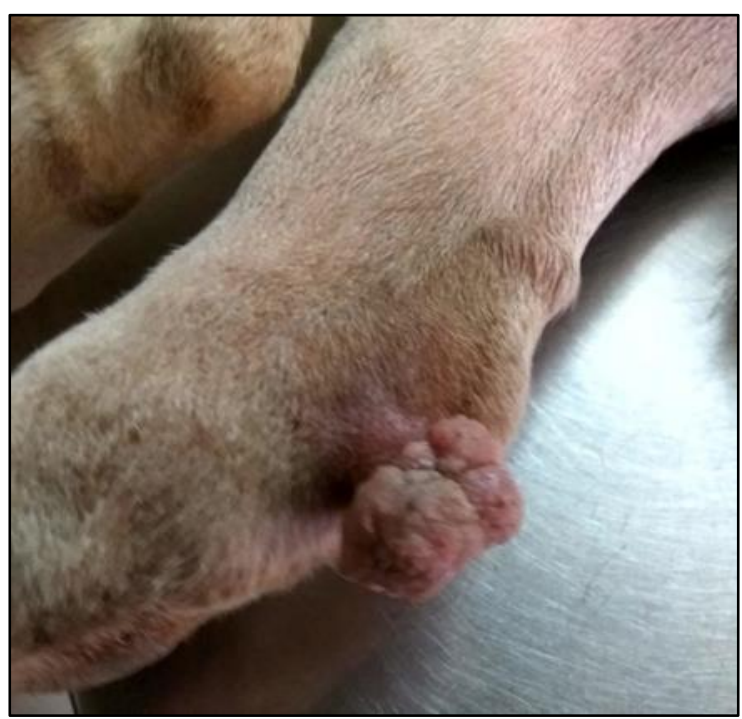

Fig 14: Cutaneous neoplasia in a geriatric dog 


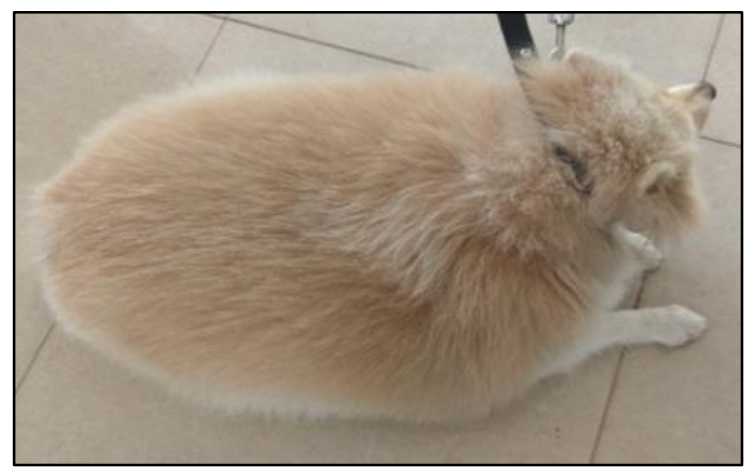

Fig 15: Obesity in a 10-year-old Pomeranian

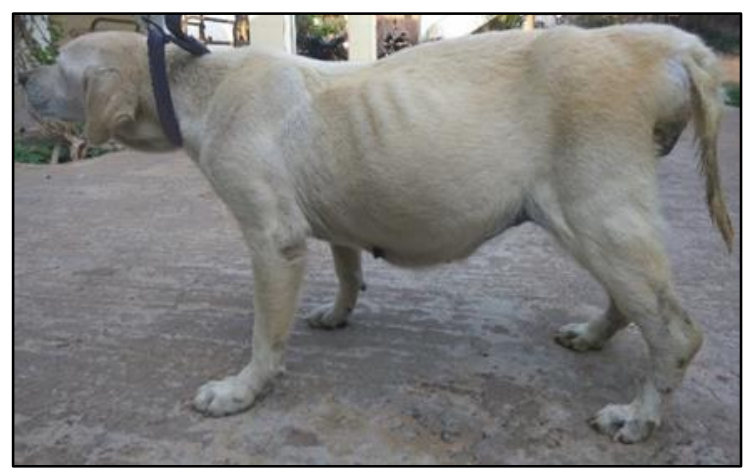

Fig 16: Pendulous potbelly abdomen in a 12-year-old Labrador Retriever

White hair is observed in 92.19 per cent of geriatric sick dogs which is due to loss of melanocytes in the hair follicles and reduced activity of the enzyme tyrosinase resulting in the production of white hairs often observed around the muzzle and face of older dogs ${ }^{[5]}$. Calluses on paw pads and elbow is observed in 76.39 per cent of geriatric sick dogs which is due to overweight, problems in joints and longer periods of lying down, deficiencies in zinc and intake of calcium supplements, which absorb zinc ${ }^{[6]}$. Loss of body weight or underweight is observed in 71.38 per cent of geriatric sick dogs which is due to alterations in whole-body protein turnover in aging resulting in muscle wasting ${ }^{7}$; age-related sarcopenia in older dogs ${ }^{[8]}$; periodontal disease like infected gums and teeth experience mouth pain, eat poorly and lose weight, kidney disease, heart disease, cancers, and loss of the senses of smell and taste or apathy ${ }^{[9]}$.

Dehydration is observed in 71.19 per cent of geriatric sick dogs which is due to azotemia, higher anion gap due to presence of lactic acid in metabolic acidosis ${ }^{[10]}$ and increased potassium and decreased renal tubular function with age ${ }^{[11]}$. Hair loss was prominent feature in 68.22 per cent of sick geriatric dogs which is due to increased cellular atrophy of epidermis and dermis, follicular atrophy resulting in areas of hair loss ${ }^{[12]}$. and old age-related nutritional deficiency ${ }^{[6]}$. Scaly skin is observed in $\mathbf{4 6 . 6 5}$ per cent and dry dull hair coat is observed in 66.36 per cent of geriatric sick dogs which is due to changes in sebum production in older animals ${ }^{[8]}$ old age-related nutritional deficiency ${ }^{[6]}$ and oil-producing skin glands don't work as efficiently ${ }^{[9]}$.

Dental abnormalities like dental tartars (60.59\%), broken tooth $(49.26 \%)$, loss of tooth $(58.36 \%)$ and plaque $(29.00 \%)$ was observed in the geriatric sick dogs of our study which is due non-maintenance of proper oral hygiene along with increased non-vegetarian diet, ${ }^{[13]}$ destruction and loss of alveolar bone along the roots of teeth, reduced jaw size, crowding of teeth, lower ratio of mandibular size to tooth volume in smaller dogs, malocclusion problems, which facilitate the deposition of subgingival plaque which is more difficult to remove and as periodontal disease progresses, there is destruction and loss of alveolar bone along the roots of teeth ${ }^{[14]}$ and plaque normally attaches daily on the crowns of teeth unless mechanically or chemically removed, calcium and phosphorus in the saliva mineralize the plaque to produce calculus ${ }^{[8]}$.

Panting is observed in 50.93 per cent of geriatric sick dogs which is due to stress, heat, pain or respiratory disease ${ }^{[15]}$. Hyperkeratosis was observed in 43.31 per cent of sick geriatric dogs which is due to loss of elasticity of skin and hair follicles which becomes less pliable as a result of increased calcium content and pseudoelastin in the elastic fibers ${ }^{[8]}$. Constipation is observed in 39.22 per cent of sick geriatric dogs which is older dogs tend to drink less water and this produces hard, dry stools that are difficult to pass and other contributing factors are lack of exercise, improper diet, reduced bowel activity, weakness of the muscles of the abdominal wall and an enlarged prostate can narrow the rectal canal and cause straining to defecate ${ }^{[9]}$.

Cutaneous neoplasia is observed in 34.57 per cent and subcutaneous mass is observed in $36.99 \%$ of geriatric sick dogs which is due to increased concentration of highly sulfated GAGs in the basement membranes of hair follicles with age ${ }^{[8]}$. Loss of vision is observed in 34.39 per cent of geriatric sick dogs which is due to development of nuclear (lenticular) sclerosis, which appears as bilateral bluish-gray haziness in the nucleus of the lens, ${ }^{[16]}$ compression of existing lens fibers due to new fiber formation, increased density of the lens, increased refractive index of lens nucleus ${ }^{8}$ and retinal diseases, glaucoma, and uveitis ${ }^{[9]}$.

Halitosis is observed in 31.59 per cent of geriatric sick dogs which is due to bacterial degradation of urea to ammonia [17] and increased blood urea nitrogen levels and reduced salivary flow rates ${ }^{[18]}$. Diarrhoea is observed in 31.23 per cent of geriatric sick dogs which is due to kidney, liver disease, pancreatic disease, malabsorption syndrome, parasites especially whipworms or cancer ${ }^{[9]}$. Loss of hearing is observed in 27.32 per cent of geriatric sick dogs which is due to cochlear degeneration ${ }^{[19]}$. Obesity is observed in 23.79 per cent of geriatric sick dogs which is due to reduced physical activity, reduced basal metabolic rate, increased fat content, decreased lean tissue and water contents with age ${ }^{[8]}$ and overweight dogs are less likely to exercise ${ }^{[9]}$. Pendulous, potbelly abdomen observed in 23.42 per cent of sick geriatric dogs which is due to Cushing's syndrome or ascites as the result of heart or liver failure ${ }^{[9]}$.

\section{Conclusion}

The clinical approach is an -important tool in the prevention and treatment of different pathologies and should consider for careful evolution of pet's health to achieve effective intervention and better health management.

\section{Acknowledgement}

The authors are thankful to the Sri Venkateswara Veterinary University for providing facilities and funds to conduct the research.

\section{References}

1. Bartges J, Boynton B, Vogt AH et al. AAHA canine life stage guidelines. J Am Anim Hosp Assoc. 2012; 48:1-11.

2. Metzger FL. Senior and geriatric care programs for 
veterinarians. Vet Clin North Am Small Anim Pract. 2005; 35:743-753.

3. State of pet health website. State of pet health 2013 report, Banfield Pet hospital. In: 2013, http://www.stateofpethealth.com/state-of-pet-health. Accessed, 2016.

4. Epstein M, Kuehn NF, Landsberg G et al. AAHA senior care guidelines for dogs and cats. J Am Anim Hosp Assoc. 2005; 41:81-91.

5. Case LP, Carey DP, Hirakawa DA, Daristotle L. Geriatrics. In: Canine and feline nutrition, (Mosby, St. louis, USA), 2000, 275-299.

6. Foster, Smith. Common diseases of older (senior,geriatric) dogs. Veterinary and aquatic services department. www.peteducation.com. 2008. Last accessed on 06-01-2015.

7. Hayek MG, Davenport GM. Nutrition and aging in companion animals. Journal of Anti-Aging Medicine. 1998; 1(2):117-123.

8. Bellows J, Colitz CM, Daristotle L, Ingram DK, Lepine A, Marks SL. Common physical and functional changes associated with aging in dogs. J Am. Vet. Med. Assoc. 2015; 246(1):67-75.

9. Eldredge DM. Dog Owner's Home Veterinary Handbook. Book production by Wiley Publishing, Inc. Composition Services. Wiley Publishing, Inc. geriatric chapter, 2007, 9.

10. Radakovich LB, Pannone SC, Truelove MP, Olver CS, Santangelo KS. Hematology and biochemistry of agingevidence of "anemia of the elderly" in old dogs. Vet. Clin. Path. 2017; 46(1):34-45.

11. Lowseth LA, Gerlach RF, Gillett NA, Muggenburg BA. Age-related changes in the prostate and testes of the beagle dog. Vet. Path. 1990; 27(5):347-353.

12. Muller GH, Kirk RW, Scott DW. Plasma cell pododermatitis. Small Animal Dermatology. 3rd ed. Philadelphia: WB Saunders Co, 1983, 713-714.

13. Ranjan R, Zahid UN, Gupta DK, Bansal BK, Dua K. An Epidemiological study on Periodontal Diseases in DogsA Clinical Study of 103 Canine Patients. Intas Polivet. 2010; 11(2):274-277.

14. Gioso MA, Shofer F, Barros PSM, Harvey CE. Mandible and mandibular first molar tooth measurements in dogs: relationship of radiographic height to body weight. J Vet. Dent. 2001; 18(2):65-68.

15. Davies M. Geriatric screening in first opinion practiceresults from 45 dogs. J Small Anim. Prac. 2012; 53(9):507-513.

16. Davidson MG, Nelms SR. Diseases of the canine lens and cataract formation. Vet. Ophthal. 2007; 2:859-87.

17. Queau Y. Gastrointestinal complications of uremia. mark acierno, dvm, dacvim (saim) Associate Professor School of Veterinary Medicine Louisiana State University Baton Rouge, LA, 2012, 74.

18. Keles M, Tozoglu U, Uyanik A, Eltas A, Bayindir YZ, Cetinkaya R. Does peritoneal dialysis affect halitosis in patients with end-stage renal disease? Peritoneal Dialysis International. 2011; 31(2):168-172.

19. Shimada A, Ebisu M, Morita T, Takeuchi T, Umemura T. Age-related changes in the cochlea and cochlear nuclei of dogs. J Vet. Med. Scien. 1998; 60(1):41-48. 